ÉGYPTE monde arabe

\section{Égypte/Monde arabe}

12-13 | 1993

Une économie en transition

\title{
Il n'y a pas de sédition confessionnelle dans notre pays
}

Al-Cha'b, 18 et 25 septembre, 2 octobre 1992

\section{Gamal al-Banna}

Traducteur : Mona Akouri

\section{(2) OpenEdition}

\section{Journals}

Édition électronique

URL : https://journals.openedition.org/ema/1306

DOI : 10.4000/ema.1306

ISSN : 2090-7273

\section{Éditeur}

CEDEJ - Centre d'études et de documentation économiques juridiques et sociales

\section{Édition imprimée}

Date de publication : 31 mars 1993

Pagination : 294-306

ISSN : 1110-5097

\section{Référence électronique}

Gamal al-Banna, « II n'y a pas de sédition confessionnelle dans notre pays », Égypte/Monde arabe [En ligne], 12-13 | 1993, mis en ligne le 08 juillet 2008, consulté le 07 juillet 2022. URL : http:// journals.openedition.org/ema/1306 ; DOI : https://doi.org/10.4000/ema.1306

Ce document a été généré automatiquement le 7 juillet 2022.

Tous droits réservés 


\title{
Il n'y a pas de sédition confessionnelle dans notre pays
}

Al-Cha'b, 18 et 25 septembre, 2 octobre 1992

\author{
Gamal al-Banna \\ Traduction : Mona Akouri
}

1 Rien, sans doute, n'illustre mieux la loi de Gresham ${ }^{1}$ - selon laquelle « la mauvaise monnaie chasse la bonne » - que le proverbe : « La faute notoire est plus prospère que l'obscure droiture », sinon ce terme de « sédition confessionnelle » qui apparaît à tout bout de champ dans le moindre écrit.

2 L'expression « sédition confessionnelle » laisse entendre qu'il y aurait dans chaque ville égyptienne, ou dans la plupart d'entre elles, des combats où s'opposent deux confessions, où coule le sang et se déchaînent les tirs, des combats qui interrompent le train de la vie quotidienne et déchirent l'unité nationale... C'est ce dont veut effectivement nous convaincre certain journal d'opposition ${ }^{2}$ qui a fait de la sédition confessionnelle son commerce favori et qui, chaque mercredi, s'orne de manchettes propres à jeter l'épouvante, semant leurs présages de malheur, de ruine et de calamités à venir... Les allégations du gouvernement et du ministère de l'Intérieur s'y trouvent enflées, décuplées; et ce journal tient sur les coptes un discours qui ne mériterait pas leur attention.

3 L'Égypte, qui s'étend sur près d'un million de $\mathrm{km}^{2}$, compte plus de 50 millions d'habitants, écoliers, étudiants, travailleurs, paysans, fonctionnaires, artisans... Tous vivent en paix. On n'entend pas parler de bagarres ayant pour origine l'appartenance ethnique ou confessionnelle... Ni à plus forte raison de "sédition». Les journaux paraissent, les salles de cinéma et de théatre donnent leurs spectacles, la télévision présente ses feuilletons...

4 Certes, quelques troubles ont eu lieu dans une zone limitée de Haute-Égypte. Plus précisément, dans deux villages connus depuis longtemps pour être le théâtre de divers démêlés. Ces problèmes, auxquels le gouvernement n'a apporté que de faux remèdes, n'ont revêtu un aspect confessionnel que lorsque s'en sont mêlés des éléments qui, incités par divers facteurs, avaient intérêt à ce qu'advienne un tel conflit - comme, sans 
doute, tel journal prétendant défendre l'entente nationale. Des litiges qui opposent deux ou trois villages - sur les quatre mille qui vivent en paix -, cela arrive dans tous les pays. Mais ces litiges n'auraient jamais été désignés sous le terme de «sédition confessionnelle » si l'on ne vivait une ère d'abus, de falsification et d'imposture, où les phénomènes sociaux sont imputés à d'autres causes que celles dont ils relèvent. La duperie a atteint son point culminant lorsque le parti au pouvoir n'a trouvé, pour se laver de sa turpitude, d'autre solution que de feindre la bienveillance à l'égard de la "glorieuse révolution du 23 juillet »... C'était tomber de Charybde en Scylla, car tout cela n'est qu'un tissu de mensonges... S'il s'agissait réellement d'étudier avec objectivité ce phénomène dit de "sédition confessionnelle ", il faudrait :

- prouver que rien, dans l'islam ou dans le christianisme, n'est susceptible de soulever les musulmans contre les chrétiens et réciproquement, puisqu'il n'existe pas de base de discorde relative aux « fondements » ou à la « doctrine » entre les deux communautés ;

- prouver que les rapports entre Égyptiens musulmans et coptes se sont caractérisés, à travers les siècles, par la fraternité et la non discrimination; que les musulmans n'éprouvent pas de susceptibilité particulière à l'égard des coptes et que ces derniers ont vécu en paix avec leurs frères musulmans : il n'y a aucune base « historique » de discorde entre les deux communautés.

\section{Islam et christianisme sont innocents du péché d'intolérance}

5 L'islam est la dernière des religions révélées. Elle a donc dû définir sa position à l'égard des religions qui l'ont précédée, ce qu'elle a fait très explicitement dans de nombreux versets coraniques et textes de la tradition qui ont tous pour objet une vérité universelle ${ }^{3}$, à savoir la reconnaissance de toutes les religions révélées, la vénération de tous les prophètes, l'affirmation que ces religions ont été révélées par le Très-Haut et que leur essence est une, essence qui s'exprime par la foi en Dieu, en les prophètes et en le Jugement dernier. Le principe d'équité dans l'islam a été poussé au point de considérer qu'il n'y a pas de différence entre ces religions. Parmi les nombreux versets sur ce thème, nous citerons ${ }^{4}$ :

Dites: «Nous croyons en Dieu et en ce qui est descendu sur nous, en ce qui est descendu sur Abraham, Ismaël, Isaac, Jacob, les Lignages, en ce qui fut donné à Moïse, à Jésus, fut donné aux prophètes de la part de leur Seigneur. De tous ceux-là, nous ne séparons pas un seul, puisque c'est à Lui que nous nous soumettons. »

C'est nous qui t'avons fait révélation, comme nous l'avions fait à Noé, aux prophètes d'après lui, fait à Abraham, à Ismaël, à Isaac, à Jacob, aux Lignages, à Jésus, Job, Jonas, Aaron, Salomon.

Et nous donnâmes les psaumes à David et à des envoyés parmi lesquels il en est de qui nous te narrâmes l'histoire, et d'autres de qui nous ne l'avons pas fait...

Et Dieu parla à Moïse [...]

Quant à la règle qui gouverne les relations entre musulmans et non musulmans, nous la trouvons dans les versets suivants :

Dieu ne vous a pas interdit ceux qui ne vous combattent pas pour cause de religion, ni ne vous évincent de votre habitat, de vous montrer envers eux vertueux, équitables : Dieu aime les équitables.

Il interdit seulement ceux qui vous combattent pour cause de religion, vous évincent de votre habitat ou concourent à votre éviction, de pactiser avec eux. Qui pactise avec eux... ceux-là sont les iniques. 
7 Tout ce qui, dans le Coran, fait allusion au conflit concerne cette communauté qui a ouvert les hostilités.

Dès l'époque du Prophète, des relations cordiales s'instauraient entre musulmans et chrétiens ; cela commença lorsque celui-ci fit partir le premier groupe de réfugiés pour l'Abyssinie "où, leur dit-il, se trouve un roi chez lequel personne n'est maltraité.» AlNagâchi ${ }^{5}$ les prit sous sa protection, eut pour eux tous les égards et refusa de les livrer à Qoraych lorsqu'on le lui demanda. Leur séjour dans ce pays dura donc dix ans et lorsqu'ils prirent le chemin du retour, al-Nagâchi les fit accompagner par une escorte chargée de les faire parvenir sains et saufs à destination. Le Prophète tint à présenter lui-même ses respects à l'escorte. Ses amis lui ayant dit : "C'est assez de nous, ô Envoyé de Dieu ", il leur répondit : "Ils ont traité nos amis avec honneur et je désire les récompenser »... Cette marque d'affection est confirmée dans le verset suivant :

Sûr que la haine la plus farouche envers les croyants, tu la trouves chez les Juifs et chez les associant; et la plus proche affinité avec les croyants, chez ceux qui se qualifient de Chrétiens : c'est qu'il y a parmi eux des pasteurs et des ermites, et qu'ils sont sans superbe.

9 L'Égypte et les coptes ont acquis la place d'honneur dans le Coran par l'intermédiaire de cet exemple donné aux croyants :

Dieu use quant aux croyants de la semblance de la femme de Pharaon quand elle dit: «Seigneur, bâtis-moi près de Toi une demeure au Paradis, et sauve-moi de Pharaon et de ses oeuvres. Sauve-moi d'un peuple d'iniquité ».

Et Marie fille de Joachim. Elle sut fortifier son sexe. Nous y insufflâmes de Notre esprit. Elle avéra les paroles de son Seigneur et des Écritures. Dévote fut-elle entre tous.

10 Cet exemple se rapporte tout entier aux coptes parce que ce sont eux les Égyptiens et ce sont eux les chrétiens.

Le Prophète s'est uni aux coptes par alliance ; nous chantions, tout enfants, comme s'il se fût agit d'un " chœur ", en évoquant les fils du Prophète.... " et tous ont été enfantés par Khadija, sauf Ibrahim qui le fut par Marie la Copte ».

L'Envoyé de Dieu considérait tous les prophètes comme des frères ; il a recommandé l'Égypte et ses coptes et il a dit que toutes les religions étaient comparables à une maison unique à laquelle il ne manquerait rien, s'il n'y avait eu cette faille que l'islam est venu combler.

13 Ces versets et textes de la tradition extirpent toute intolérance, sèment l'amour et la fraternité dans les coeurs, font grand cas de l'Égypte et de ses coptes ; eux-mêmes sont les premiers à le savoir et à s'en prévaloir car cela constitue une reconnaissance de leur identité et un témoignage du respect qui leur est voué.

14 Le christianisme a lui aussi, de son côté, défini sa position par rapport aux religions qui existaient avant qu'il ne fût révélé ; définition valable également pour l'islam qui lui est postérieur, du fait surtout que les religions contemporaines du christianisme étaient païennes, à l'exception du judaïsme. Malgré cela, le christianisme fut conciliant avec elles et exigea des chrétiens la même tolérance.

15 À deux occasions significatives, deux hommes d'église ont exposé la position du christianisme à l'égard d'autres religions en vigueur: d'abord en 1919, lorsqu'une vague de fraternité a soudé les deux communautés musulmane et chrétienne en réplique aux tentatives des Anglais pour semer division et discorde. Mgr Basileus Mûsa, représentant des coptes catholiques d'Égypte, avait alors tenu plusieurs conférences, 
réunies depuis dans un petit volume intitulé al-Din wa-l-wataniyya (Religion et Patriotisme), édité au Caire en 1920. Puis ce fut lors de la crise qui opposa le président Sadate au chef de l'Église copte. Le père Matta al-Miskin ${ }^{6}$ avait alors proclamé son opinion dans un long entretien avec Makram Muhammad, publié dans al-Ahrâm du 17/4/1980 sous le titre : "Qirâ'a fi Awrâq al-Abb Matta al-Miskin » (Lecture des feuilles du père Matta al-Miskin) et que nous citons dans notre ouvrage "Al-Hasasiyya al-Dîniyya " (Les susceptibilités religieuses).

\section{Patriotisme et religion ne sont pas antagonistes}

Mgr Basileus a abordé la question en développant deux points :

- le premier est centré sur l'idée que la religion nous commande d'aimer et de servir la patrie malgré la diversité des appartenances religieuses de nos compatriotes : le patriotisme est une chose et la religion en est une autre, d'où l'absence de tension entre ces deux catégories ;

- le deuxième point nie l'existence d'une raison qui légitimerait le laxisme religieux par égard pour la patrie et affirme qu'un tel laxisme porterait un coup mortel au vrai patriotisme.

Dans le premier point, l'auteur indique que religion et patriotisme sont deux choses différentes, non antagonistes, à moins que les deux concepts soient mal compris ou qu'on prémédite de leur porter atteinte. Il ajoute qu'avant le christianisme, les gens ne faisaient pas de distinction entre le spirituel et le temporel, entre la religion et la patrie, qui se fondaient en une seule et même entité. C'est cette croyance qu'exprime le célèbre proverbe latin qui dit en substance que la condition essentielle de l'appartenance à une patrie, c'est d'adorer ses dieux.

Pour Mgr Basileus, un tel principe détruit la liberté de conscience et engage les conquérants à faire en sorte que les vaincus se convertissent à leur religion. Les Romains n'ont pas fait exception à la règle bien qu'ils aient maquillé leur politique par la ruse; en effet, tout en permettant aux populations des pays conquis de conserver leurs dieux, ils les obligeaient à adorer également les dieux de Rome. Avec la venue du christianisme, qui annonçait le rejet de tous les dieux païens, les Romains considérèrent les chrétiens comme des incroyants, des traîtres, non seulement envers la patrie mais aussi envers tout le genre humain car à cette époque, Rome prétendait représenter la civilisation et justifiait, par ses accusation, l'oppression qu'elle exerça contre les chrétiens.

Ceux-ci disaient :

«Ô État de Rome! Il est vrai que nous n'adorons pas tes dieux et que nous ne pouvons les adorer; sache cependant que, malgré cela, nous sommes ceux qui te sont le plus loyalement dévoués, car la religion est autre que l'État: elle est du ressort de la conscience, alors que l'État doit son édification à des conditions géographiques naturelles ou à des victoires, et n'a en tout cas rien à voir avec ce qui relève de la conscience. »

20 L'auteur attire l'attention sur le fait que, malgré l'augmentation du nombre de chrétiens et l'imprégnation de tous les milieux et de tous les modes de vie par le christianisme, il n'était pas venu à l'idée de cette multitude de se soulever un jour contre l'État ou contre ses rois.

21 À l'oppression, les chrétiens opposèrent la patience, et le sang de trois générations coula sans qu'il y eut de protestation. C'est une preuve incontestable du fait que, 
parallèlement à leur attachement au christianisme, ils étaient loyaux envers leurs souverains alors que ceux-ci ne leur épargnaient ni tourment ni torture.

Mgr Basileus évoque enfin à juste titre le fait qu'il n'y avait pas de recrutement obligatoire dans l'armée romaine, et que les chrétiens s'y enrôlaient cependant comme volontaires et en grand nombre pour se battre héroïquement sous les drapeaux frappés de l'aigle sans se départir de leur attachement à leur religion. L'auteur cite pour finir de nombreux exemples dans lesquels l'Empereur ou les chefs romains avaient voulu contraindre des légions et des groupes de soldats chrétiens à offrir des sacrifices aux dieux de Rome et ne rencontraient que le refus.

Il incombe au chrétien de se soumettre, en conscience, au pouvoir séculier légitime, comme le lui prescrit un passage de l'Epître de saint Paul aux Romains. Or, à l'époque, ces derniers étaient gouvernés par Néron, ennemi des chrétiens et du christianisme ; la folie l'avait égaré au point de le pousser à incendier Rome pour pouvoir imputer ce crime aux chrétiens puis les exterminer après avoir monté l'opinion contre eux. Et Paul semblait alors leur dire : "O chrétiens de Rome! Vous n'avez pas d'ennemi pire que Néron, mais puisque le pouvoir légal est entre ses mains, il est de votre devoir, en conscience, de lui obéir. $»^{7}$

Saint Paul enjoignait à Tite de rappeler au peuple qu'il doit «être soumis aux autorités » (Epître à Tite, 3,1 ). Son enseignement allait se perpétuer à travers Pierre, le principal apôtre, qui disait: "Soumettez-vous à toute institution humaine à cause du Seigneur : soit au roi, comme souverain, soit aux gouverneurs, comme envoyés par lui pour châtier les malfaiteurs et louer les gens de bien. » (Première Epître de Saint Pierre 2,13-14)

«Une fois apportés ces preuves et indices clairs, peut-on encore, se demande Mgr Basileus, douter du fait que la distinction entre religion et patrie trouve ses origines dans le christianisme?»

Les apôtres savaient que la loyauté absolue à l'égard du pouvoir temporel était un devoir et saint Paul ordonna « que soient récitées oraisons, prières, invocations, actions de grâce pour tous les gens, pour les rois et tous ceux qui détiennent un pouvoir, afin que l'existence se déroule en paix. Ceci est bon et agréé par Dieu notre Sauveur ».

Ainsi l'Église, se conformant aux instructions de l'apôtre Paul, n'a jamais manqué de demander à Dieu, dans ses prières officielles, de protéger les rois et de renforcer leurs alliances, même si ces souverains ne sont pas chrétiens, voire même ennemis de la chrétienté. Aussi, de la première génération jusqu'à aujourd'hui, l'Église copte n'a pas un seul jour omis de prier, au cours de la sainte messe (le plus sublime de ses rites) pour les détenteurs du pouvoir, alors que dès la septième génération, l'Égypte n'était plus gouvernée que par des non chrétiens, dont certains ont maltraité les chrétiens.

\section{Entretien avec le père Matta al-Miskin}

Le point de vue du christianisme s'est précisé pour la seconde fois lors de la dégradation des relations entre le président Sadate et le chef de l'Église copte: le journaliste Makram Muhammad Ahmad a alors recueilli les propos du Père Matta alMiskin dans un entretien publié à l'époque par le journal Al-Ahrâm, dans lequel on pouvait lire : «Le moine Matta al-Miskin, doyen du couvent de Wadi-l-Natroun et qui vit dans le dénuement, a fait preuve d'une grande clarté tout au long de cet entretien, 
notamment en expliquant que l'Église a une mission précise qu'elle ne doit jamais outrepasser : sauver les âmes en prêchant la Bonne Nouvelle et la Parole de Dieu. De son point de vue, l'Église, même en s'adonnant à des oeuvres sociales, dépasse les limites fixées par sa mission, se fourvoie, s'engage dans des situations incompatibles avec son rôle."

$\mathrm{Au}$ journaliste qui lui demandait de préciser quelles formes de rapports devrait entretenir l'Église avec la société, il a répondu :

«L'Église a pour mission, non de servir la société mais de servir la Foi, le Christ, à travers les pécheurs, les déshérités, les humiliés, les sans-abri; chaque fois que l'Église a dérogé aux prescriptions du Christ, qu'elle s'est laissé tenter par le pouvoir temporel, qu'elle a attisé les passions au nom de la Croix, qu'elle a dévié de sa voie pour aller vers l'argent et l'influence, et tenté de concilier pouvoir religieux et pouvoir temporel, chaque fois, enfin, qu'elle a revendiqué avec zèle des droits communautaires ou ethniques, elle a échoué à faire passer le message du Christianisme et a vu se propager dans ses rangs rivalités, dissensions et faiblesse, se fermer devant elle les portes du Royaume des Cieux. Elle s'est retrouvée dans la nécessité de trouver un sauveur pour la tirer de ce mauvais pas et la rétablir dans son rôle initial. »

Matta al-Miskin s'est imposé des limites rigoureuses : il perçoit comme une infraction les oeuvres sociales accomplies par l'Église car, dit-il,

«l'assistance sociale suppose que l'on assure le patronage des jeunes et leur orientation, l'instruction des ouvriers et l'évaluation de leur situation et de leurs revendications, la prise en charge des étudiants, l'établissement de clubs de jeunesse, l'organisation de camps, de débats sur les problèmes de la jeunesse, la construction d'hôpitaux, d'hospices... Autant d'activités qui relèvent des autorités. Et sachant que tout régime a nécessairement son orientation propre, ses plans de gestion et d'assistance auprès de toutes les couches sociales, il paraît indispensable que l'Église ait acquis une connaissance dudit régime afin que ses projets soient conformés à ceux du gouvernement ; faute de quoi le conflit entre l'Église et l'État est inévitable. »

31 Où se situe donc la ligne de démarcation entre le pouvoir de l'Église et celui de l'État? Matta al-Miskin répond avec une rare précision :

«Il n'existe ni contact ni frontière entre les deux, car le Royaume de l'Église, c'est le Royaume de Dieu, alors que celui du souverain, c'est le monde, c'est le royaume d'ici-bas, c'est notre vie quotidienne et notre organisation sociale; ce qui est à César revient à César et ce qui est à Dieu revient à Dieu. Telles sont les frontières que définit la parole du Christ. »

Répondant à notre désir d'éclaircissement, le Père Matta al-Miskin ajoute :

«Si l'Église est incapable d'entretenir la Foi par la persuasion et l'amour et qu'elle s'empresse d'aller vers les rois et chefs d'État pour leur faire promulgue un édit en ce sens, elle aura fait fausse route : ce n'est ni l'épée ni la loi qui peuvent garantir la Foi ; celle-ci trouve son secours dans le prêche de la Bonne Nouvelle, de la Parole de Dieu.

Lorsqu'au IV ${ }^{e}$ siècle, l'Église a demandé protection au roi Constantin, le priant de défendre la Foi par l'épée, qu'est-il arrivé ? Constantin a mené sa croisade de par le monde, levant la bannière portant la Croix au-dessus des propagateurs d'inimitié, transformant le symbole même de la vie en un symbole de mort. Ce n'était pas de combattre ses ennemis qui était infâmant : c'était qu'il le fît au nom de la Croix.

Que l'Église ait recours à la force du pouvoir séculier ou qu'elle incite à n'en pas tenir compte sont deux choses équivalentes; dans la première, elle outrepasse ses compétences; dans la deuxième, elle transgresse la volonté du Christ, se rend coupable d'ingérence dans les desseins de Dieu. Inciter au mépris de l'autorité de 
l'État, qui représente le pouvoir temporel, revient à inciter au mal car l'Église ne doit jamais adopter de position hostile vis-à-vis de l'État ou de la patrie ; or, ceux qui devraient enseigner la religion sèment la discorde, la division, le sectarisme.

Jésus-Christ a dit : «Il n'est de pouvoir que par Dieu " $^{8}$ et « Rendez à tous ce qui leur est dû : l'impôt à qui il est dû, et les taxes de même ; la crainte et l'honneur à qui ils sont dus. $»^{9}$ Par conséquent, la conduite du citoyen chrétien, en ce qui concerne le pouvoir temporel, n'est pas du ressort de l'Église. Celle-ci n'est pas en mesure - ni n'est censée l'être - de demander des comptes à un ministre ou un responsable chrétiens de leurs agissements au sein du gouvernement, car en ce domaine, ils ne relèvent pas de son autorité. Quant au citoyen chrétien, elle ne peut lui demander de comptes qu'en ce qui concerne sa foi et son attitude spirituelle; ceci est pour lui une garantie de sa liberté d'action, d'opinion, de participation à toute activité civique ou sociale, sans que l'Église ne soit considérée comme responsable du manquement éventuel de ses membres à leur devoir national, de leur engagement dans telle ou telle voie ou de leur attitude vis-à-vis du pouvoir [...]. »

Matta al-Miskin dit en substance que le patriotisme du chrétien et toutes les conduites particulières et générales qui s'y rattachent sur les plans social, économique ou politique ont leur origine dans l'entité du citoyen et non dans celle de l'Église ; c'est l'État qui est concerné par le patriotisme du citoyen chrétien, dont il est, lui, responsable, et non l'Église ni les religieux.

La voix de Matta al-Miskin nous parvient de sa cellule de Wadi-l-Natroun, douce, recueillie et claire ; elle nous avertit : son dessein est de sauver l'Église du danger qui la menace si elle oublie sa vocation initiale, si elle oublie que l'ennemi qui menace le plus de la réduire à néant, c'est la préoccupation de ses dignitaires pour autre chose que le péché de l'homme, la négligence de leur rôle - communiquer l'appel du Christ aux pécheurs; pour Matta al-Miskin, ceci constitue non seulement une dérogation aux règles du christianisme, mais aussi un acte de rébellion.

De son point de vue comme de celui de l'apôtre Paul, la question est grave, car « ceux qui tentent de détourner l'Église de sa mission le font par incapacité à prêcher la contrition qui doit renouveler l'homme et le racheter; la perte que subira l'Église par ces innovations dans les thèmes de prédication aboutira à l'extinction de cette lumière qu'est l'appel au repentir pour la rédemption des pécheurs.

Les prédicateurs ont quitté le chemin qui mène au coeur de l'homme et se sont égarés dans un dédale de questions tout à fait éloignées de la vocation de l'Église [...]. Ils oublient que le Christ n'a jamais confondu le Royaume de Dieu et celui d'ici-bas; s'occuper de ces questions temporelles au nom du Christ, c'est comme vouloir le consacrer roi de la terre; de même, renforcer le pouvoir de l'Église sous prétexte de revendiquer les droits de la communauté constitue une nouvelle tentative pour instaurer le royaume du Messie tel qu'en rêvent les juifs.

Si l'Église purifie sa pensée de toute ambition terrestre, renonçant à parler de droits requis et de droits usurpés, elle se souviendra alors de la parole de son Maître : "Mon Royaume n'est pas de ce monde $»^{10}$; elle se souviendra qu'elle doit se consacrer entièrement au repentir car telle est sa mission. Si l'on niait à l'Église sa compétence dans l'appel à la contrition du fait de ses préoccupations temporelles, elle se retrouverait sans rôle, car à l'origine, le fondement de son oeuvre était déjà de «parfaire le repentir afin de garantir le salut.»

$38[. .$.$] « En résumé, il n'existe pas, pour l'Église, d'autre tâche que la pénitence [...].$ 
La délicate et fragile voix qui, depuis Wadi-l-Natroun, nous parle à tous, musulmans et chrétiens, c'est la voix éternelle de l'ermite égyptien qui s'est fait entendre tout au long de l'histoire de l'Église et nous parvient aujourd'hui par la bouche de Matta al-Miskin, porteuse non seulement d'une mise en garde collective contre un débordement, par l'Église, des limites de sa mission, mais aussi d'une consigne précise. Elle nous dit, avec un courage chrétien profondément riche et fécond:

«Toute tentative pour associer le Royaume de Dieu, aboutissement d'une vocation qui se régénère, à d'autres desseins, tels que la revendication, pour l'Église, de droits particuliers - participation au gouvernement ou à la gestion des affaires de l'État, droit d'accéder à des honneurs terrestres, influences, souverainetés... constitue une déviation de l'objectif auquel tend la mission de l'Église : accéder au Royaume de Dieu ».

De son point de vue, la question est d'une extrême simplicité :

«Si l'Église dévie de l'objectif défini par sa vocation [...], à quelle source puisera-telle pour couvrir ses frais? L'argent indispensable, d'où viendra-t-il ? De la vente des dons divins, des secours implorés à l'intérieur comme à l'extérieur? Alors que si elle s'en tenait à son but réel, elle investirait ses forces dans les prédications désintéressées, bénévoles, et resterait unie aux pécheurs assoiffés de pénitence. »

Il apparait, à travers cet exposé, qu'islam et christianisme sont au-dessus de tout soupçon en matière de fanatisme et qu'il n'existe rien, dans leurs références - Coran, Sunna ou Évangiles - pouvant dresser une religion contre une autre; toutes deux, quelles que soient leurs différences, prêchent la charité et l'amour entre tous les hommes.

\section{Le verdict de l'Histoire}

Si rien, tant dans les fondements de l'islam que dans ceux du christianisme, ne suscite de discorde, existe-t-il par ailleurs, dans l'histoire des relations entre musulmans et coptes, quelque précédent susceptible de justifier pareilles tensions?

La relation entre l'islam et les coptes débute avec l'entrée de Amr Ibn al-'As en Égypte (en 639 de l'ère chrétienne; $n d l r$ ) : il libère les coptes de la domination des Romains et de l'évêque qui leur avait été imposé à la place du patriarche Benyamin, obligeant ce dernier à se réfugier dans le désert. Le conquérant proclame la liberté religieuse, rétablit le Pape Benyamin dans son rôle avec tous les égards qui lui sont dus et lui rend tous ses pouvoirs sacerdotaux: la conquête de l'Égypte par Amr Ibn al-'As est une libération pour les chrétiens et la religion copte.

Certes, au cours des mille trois cents ans d'hégémonie islamique, les coptes ont subi l'oppression de certains gouverneurs, mais celle-ci ne s'exerçait pas spécifiquement contre eux : elle touchait également les musulmans. En outre, certains jurisconsultes ont propagé une conception erronée quant à la manière dont on traitait les sujets non musulmans ${ }^{11}$, surtout en ce qui concerne la perception de la jiziyya ${ }^{12}$ : il est des pratiques d'exception qui ne peuvent en aucune manière être mises sur le compte de l'islam, le Coran et la Sunna étant ses seules références.

L'attention de certains s'est en effet fixée de manière obsessionnelle sur le terme de jiziyya : ils se sont mis à dénoncer cette "imposition » et à en faire grief à l'islam comme si elle lui était spécifique. Or, le tribut imposé au pays conquis est une pratique en vigueur depuis les temps les plus reculés ; l'islam n'a fait que la reprendre après y avoir 
apporté des modifications destinées à en éliminer les imperfections, de sorte que la jiziyya puisse constituer un substitut la conscription ; car les non musulmans auraient pu trouver quelque inconvénient à être enrôlés dans les rangs de l'armée musulmane ; c'est pourquoi femmes et enfants en étaient dispensés.

Le tribut dans ses pires formes était connu des Romains, et saint Paul avait ordonné aux chrétiens de le payer :

«Voilà bien pourquoi vous payez les impôts ; car les magistrats sont des servants de Dieu assidus à leur office. Rendez à tous ce qui leur est dû : à qui l'impôt, l'impôt ; à qui les taxes, les taxes ; à qui la crainte, la crainte ; à qui l'honneur, l'honneur. »13

\section{L'Histoire du patriarche Korollos}

Nous n'avons pas besoin d'aller chercher très loin dans l'Histoire pour déterminer la position du pouvoir islamique : Amr Ibn al'-As avait ramené le patriarche Benyamin du désert où il s'était réfugié, fuyant l'oppression romaine ; en 1892, le khédive Tawfiq fait de même avec le patriarche Korollos V.

Cependant on découvre, dans cette dernière circonstance (exil puis réhabilitation de Korollos V) qu'une relation chaleureuse liait le khédive musulman au patriarche copte et qu'ils se portaient mutuellement un profond respect.

En 1892, les relations entre le patriarche et le Conseil communautaire (majlis milli) ${ }^{14}$ étaient tendues à l'extrême et les tentatives de réconciliation avaient échoué. Le Conseil, alors présidé par Boutros Ghali Pacha ${ }^{15}$, cheikh de la communauté copte, décréta, en date du 1er septembre 1892, « l'éloignement du patriarche dans le couvent al-Baramos ${ }^{16}$, cela pour avoir contrevenu aux ordres du gouvernement et pour son désaccord avec la communauté ; rejetant les tentatives de réconciliation, il a continué à envoyer ses hommes semer l'agitation dans les provinces et à falsifier des dépêches qu'il destinait à la Cour ", etc. Ainsi sont relatés les faits dans l'ouvrage de Boutros Ibrahim, Al-ldahat al-jaliyya fi tarikh hawadith al-mas'ala al-qubtiyya, Le Caire, $1893^{17}$.

Le Conseil communautaire soumit ce décret au "conseil des ministres" (majlis alnuzzar)qui le ratifia: il fallait mettre un terme à une situation qui risquait de semer agitation et division au sein de la communauté et de troubler l'ordre public. Ordre fut donc donné au gouverneur d'Alexandrie de surveiller le départ du patriarche pour le couvent, le 8 septembre 1892 .

51 Rappelons que lorsque le train conduisant le patriarche arriva à hauteur de Damanhour, Hamza Bey, cheikh des Bédouins, l'accueillit avec ses cavaliers et l'escorta jusqu'à mi-chemin ; il ne devait abandonner le convoi que sur l'insistance du patriarche lui-même. Cette manifestation poussa un groupe du Conseil communautaire à remettre en question la mesure adoptée. Boutros Ghali réunit les deux factions à son domicile de Faggala et une délégation fut envoyée au Conseil des ministres dont elle rencontra le président, Mustapha Pacha Fahmi : elle le pria de rappeler le patriarche. La délégation fut reçue par le khédive le 28 novembre. Le journal al-Watan rapporte la rencontre comme suit :

«À trois heures, heure franque, dans l'après-midi du lundi 28 novembre, la délégation copte fut honorée de l'éminente présence khédivienne qui trouva devant elle quatre personnes: Ezzetlou Awad Allah Bey Serour, Ezzetlou Ibrahim Bey alWahabi, Hanna effendi Ghorbal et Boutros Effendi Fadl Allah; ils prièrent sa majesté de rappeler sa Béatitude le patriarche des coptes, n'ayant d'autre recours et 
appui que dans sa Grandeur le khédive, car il est leur souverain équitable qui veille à leur repos et celui qui met fin à leur dispersion et à leur division ; il les considéra Dieu l'ait en Sa sainte garde - avec affection et bonté et leur promit d'accéder à leur demande.

La délégation étant arrivée au couvent al-Baramos et ayant rencontré le patriarche, celui-ci déclara : "J'ai été éloigné de mon Siège par ordre de Sa Majesté, notre sublime khédive. Ses ordres m'ont été transmis de ne rien dire ni faire ; il ne serait pas convenable de ma part de contrevenir aux instructions de notre Souverain et Bienfaiteur. » Les membres de la délégation répondirent: « Nous sommes envoyés par sa Grandeur. » Il s'enquit : « Avez-vous entre les mains la lettre qui vous permet d'agir? Si vous êtes porteurs d'un message du gouvernement, j'obéirai immédiatement à l'ordre de sa Majesté. »

52 À la magnanimité du patriarche correspond celle du khédive. Qelini Pacha Fahmi raconte dans ses Mémoires que ce dernier avait beaucoup de respect pour le patriarche et croyait profondément en son mérite. S'il devait évoquer Sa Béatitude au cours d'une conversation, il disait : "Notre Saint Maître le Patriarche m'a dit telle et telle chose.» Qelini Fahmi lui-même a proposé que le retour du patriarche ait lieu "en grande pompe » pour que se cicatrisent les blessures provoquées par ce drame. Le khédive et Riyad Pacha Fahmi tinrent compte de toutes ses propositions.

De cet exposé de l'histoire des relations entre le pouvoir islamique et les coptes, il ressort que rien n'est susceptible de provoquer tension ni conflit, que ledit pouvoir faisait bénéficier les coptes de sa tolérance et de sa fraternité, et qu'en outre il avait pris en considération leur spécificité et permis l'accès de maints d'entre eux aux plus hautes fonctions, outre la puissance économique et financière à laquelle les coptes purent prétendre du fait de leur solidarité et de leur coopération.

\section{NOTES}

1. Gresham (Sir Thomas) : financier anglais (1519-1570). Il est notamment l'auteur de l'Enquête sur la chute du change (Ndt).

2. Il s'agit probablement d'al-Ahali, organe du parti al-Tagammu' (« Rassemblement ») de gauche.

3. «Vérité universelle » : s'entend ici dans le sens philosophique du terme, où « universel » est le contraire de « particulier » et n'admet pas d'exception.

4. Les citations coraniques sont extraites de : «Le Coran », essai de traduction de l'arabe, annoté et suivi d'une étude exégétique par Jacques BERQUE ; éditions Sindbad, Paris, 1990. Sourate II, La Vache, verset 136 ; Sourate IV, Les Femmes, versets 163 et 164 ; Sourate LX, L'Examinante, versets 8 et 9 ; Sourate V, La Table pourvue, verset 82 ; Sourate LXVI, L'Interdiction, versets 11 et 12.

5. Al-Nagâchi : roi chrétien d'Abyssinie.

6. Matta al-Miskin : ce nom signifie littéralement «Matthieu le misérable ». Considéré comme la tête de l'opposition au pape Chenouda au sein de l'Eglise copte. Vit dans un couvent à Wadi-lNatroun.

7. Toutes les citations sont tirées de la Bible d'Osty, éditions du Seuil, Paris, 1973.

8. Il s'agit en fait de Saint Paul ; cf. Epître aux Romains, 13, 1 et 7 . Tout le paragraphe qui précède est un résumé de ce chapitre 13.

9. Idem. 
10. Évangile selon St. Jean, XVIII, 36.

11. Ou « dhimis », qui signifie précisément ceux qu'« en conscience » l'islam doit protéger.

12. jiziyya : tribut que payaient les non musulmans en terre d'islam.

13. Paul, Epitre aux Romains, 13, v. 6-7.

14. Majlis milli : suivant les instructions des autorités ottomanes, la communauté copte se dota d'un conseil séculier chargé d'administrer les affaires non religieuses de la communauté.

15. Grand-père de M. Boutros Boutros-Ghali, actuel secrétaire général de l'ONU.

16. Couvent de la Vierge d'al-Baramos, dans la région de Wadi-l-Natroun.

17. «Explications et éclaircissements relatifs aux chroniques de la question copte».

INDEX

Mots-clés : al-Banna (Gamal), christianisme, islam, Coptes 